\title{
Stomatin-knockdown effectively attenuates sepsis-induced oxidative stress and inflammation of alveolar epithelial cells by regulating CD36
}

\author{
KANGKANG WU ${ }^{1}$ and $\mathrm{LI} \mathrm{WANG}^{2}$ \\ ${ }^{1}$ Department of Infectious Disease, Children's Hospital of Nanjing Medical University, Nanjing, Jiangsu 210008; \\ ${ }^{2}$ Department of Respiratory and Critical Care Medicine, The Affiliated Jiangning Hospital of \\ Nanjing Medical University, Nanjing, Jiangsu 211100, P.R. China
}

Received January 11, 2021; Accepted September 23, 2021

DOI: 10.3892/etm.2021.10992

\begin{abstract}
Sepsis-induced acute lung injury is a type of lung disease with a high fatality rate that is characterized by acute inflammation. In the present study, the underlying role and potential mechanism of the stomatin (STOM) protein were investigated in lipopolysaccharide (LPS)-induced oxidative stress and inflammation in a mouse lung epithelial cell line, MLE-12. The expression levels of STOM and CD36 were measured using reverse transcription-quantitative PCR and western blotting. Subsequently, the expression levels of STOM and CD36 in LPS-treated MLE-12 cells were knocked down or overexpressed, respectively, via transfection with a small interfering RNA-STOM or a CD36-overexpression vector. An RNA immunoprecipitation (RIP) assay was used to determine the interaction between STOM and CD36, while Cell Counting Kit-8 assay and ELISA were performed to detect cell viability and oxidative stress, respectively. Moreover, western blotting and ELISA kits were used to detect the expression levels of associated inflammatory factors. The results of the present study demonstrated that STOM expression was upregulated in MLE-12 cells treated with LPS compared with the untreated control group. According to the Search Tool for the Retrieval of Interacting Genes/Proteins database, it was predicted that STOM and CD36 had the ability to interact with each other. The predicted binding between STOM and CD36 was verified using a RIP assay. The results demonstrated that STOM positively regulated the expression of CD36. Moreover, in LPS-treated MLE-12 cells, STOM-knockdown reversed the inhibitory effects of LPS on cell viability, and the promoting
\end{abstract}

Correspondence to: $\mathrm{Dr} \mathrm{Li}$ Wang, Department of Respiratory and Critical Care Medicine, The Affiliated Jiangning Hospital of Nanjing Medical University, 168 Gushan Road, Dongshan Street, Jiangning, Nanjing, Jiangsu 211100, P.R. China

E-mail: liwanglwlw@163.com

Key words: stomatin, CD36, oxidative stress, inflammation, MLE-12 effects of LPS on oxidative stress and inflammation. These aforementioned changes were alleviated by the overexpression of CD36. To conclude, the results of the present study revealed that STOM may interact with CD36 to affect the levels of oxidative stress and inflammation in LPS-treated MLE-12 cells.

\section{Introduction}

Sepsis refers to the life-threatening organ dysfunction caused by the uncontrolled immune response of the host to infection, which is one of the main causes of death worldwide $(1,2)$. Using data from 1,553 reports from high-income countries with cases between 1979 and 2015, the researchers initially extrapolated that there are an estimated 31.5 million cases of sepsis and 19.4 million cases of severe sepsis globally, with a potential 5.3 million deaths per year (3). Acute lung injury (ALI) is one of the most common complications of sepsis. According to statistics, the fatality rate of ALI may account for $34.9 \%$ of the total number of individuals admitted to hospital; thus, ALI has attracted extensive attention from healthcare researchers (4). ALI refers to the damage of alveolar epithelial and capillary endothelial cells caused by direct and indirect factors, resulting in acute hypoxic respiratory insufficiency. The most important pathological feature of ALI is an extensive inflammatory reaction (5). Although treatments of ALI have improved in recent years, there are currently no drugs approved by the Food and Drug Administration or the China Food and Drug Administration for ALI treatment (6). Therefore, discovering therapeutic targets and drugs for the treatment of ALI and reducing the mortality rates associated with ALI have become the focus of current research.

Stomatin (STOM) protein is a unidirectional, low-polymerized lipid raft-related protein, also known as human erythrocyte integrated membrane protein (7). Its main function is to regulate the activity of ion channels, glucose transporters and cytoskeleton protein recombination $(8,9)$. Previous studies have identified that STOM is abnormally expressed in a variety of malignant tumors, such as lung cancer and oral squamous carcinoma, suggesting that it may be involved in the occurrence and development of tumors $(10,11)$. However, 
to the best of our knowledge, there are few studies that focus on the role of STOM in diseases other than tumors. Moreover, results of a previous study demonstrated that STOM expression is increased in children with sepsis, and that STOM may potentially be used as a transcriptional marker for the diagnosis of sepsis (12). In addition, the expression level of STOM is significantly increased in lung alveolar epithelial cells that have been damaged due to ischemia/reperfusion injury (13). Hypoxia leads to an increase in the levels of reactive oxygen species (ROS) and oxidative stress, which then promotes the occurrence of cellular inflammation (14). Therefore, it was hypothesized that STOM may affect inflammation and oxidative stress in sepsis-treated alveolar epithelial cells.

CD36 is a pattern recognition receptor that is expressed in various types of cells, including microglial, immune and tumor cells (15). CD36 mediates lipid uptake, immune recognition, inflammation, molecular adhesion and apoptosis (15). Results of a previous study revealed that CD36 regulates the innate immune response during pneumonia, tuberculosis, malaria, human immunodeficiency virus and sepsis in a ligand-mediated manner (16). A prior study indicated that ETS domain-containing protein Elk-1 B domain works primarily through CD36, and it reduces lung barrier dysfunction, neutrophil migration into the lung and lung inflammation caused by lipopolysaccharide (LPS) (17). Moreover, the inhibition of CD36 significantly suppresses LPS-induced inflammation and ALI (17). Thus, it is important to determine whether STOM promotes the injury of alveolar epithelial cells induced by sepsis by regulating CD36.

In the present study, the expression levels and biological functions of STOM and CD36 in the LPS-treated alveolar epithelial cell line, MLE-12, were analyzed. The results of the present study may provide a novel theoretical basis for STOM-knockdown in the treatment of sepsis-induced ALI.

\section{Materials and methods}

Cell culture and treatment. The alveolar epithelial cell line MLE-12 was purchased from the BeNa Culture Collection; Beijing Beina Chunglian Institute of Biotechnology. Cells were cultured in DMEM/F12 Coon's medium (DMEM/F12; Thermo Fisher Scientific, Inc.) containing 10\% FBS (Thermo Fisher Scientific, Inc.) and maintained in a $5 \% \mathrm{CO}_{2}$ incubator at $37^{\circ} \mathrm{C}$. After the cells were passaged three times, cells in logarithmic phase were selected for follow-up assays.

The sepsis cell model was established using MLE-12 cells. Cells in the logarithmic phase were routinely cultured for $24 \mathrm{~h}$ at $37^{\circ} \mathrm{C}$. When the degree of cell fusion reached $90 \%, 100 \mathrm{ng} / \mathrm{ml}$ LPS (Sigma-Aldrich; Merck KGaA) was added to the culture for $24 \mathrm{~h}$ at $37^{\circ} \mathrm{C}$ based on data from a previous study (18).

Bioinformatics analysis. Microarray data were obtained from the GEO database (www.ncbi.nlm.nih.gov/geo). Gene expression profiles of the neonatal sepsis samples public dataset GSE145227 was downloaded from GEO (https://www. ncbi.nlm.nih.gov/geo/query/acc.cgi) (19). The GSE145227 dataset contained 10 samples from children with sepsis and 12 samples from healthy controls. Affymetrix Human IncRNA Array (version 1.0; Affymetrix; Thermo Fisher Scientific, Inc.) was used to detect the expression levels of long non-coding (lnc)RNAs and mRNAs in both septic and control groups. Subsequently, the data were analyzed using GEO2R (https://www.ncbi.nlm.nih.gov/geo/geo2r) and GraphPad Prism 8.0 software (GraphPad Software, Inc.). Search tool for recurring instances of neighboring genes (STRING) database (version 11.0; https://version-11-0.string-db.org/) was used to analyze the potential association between STOM and CD36.

Cell transfection. Small interfering si(RNA) against STOM (si-STOM; si-STOM-1, $1 \mu \mathrm{g}$, 5'-GTGTTTCTAAAGATG GAATTTCA-3'; si-STOM-2, $1 \mu \mathrm{g}$, 5'-GAGTCATCTATT CTGATTATTTG-3') and the scrambled negative control group (si-NC; $1 \mu \mathrm{g} ; 5$ '-ACTTGCGCTTGCGAAAATCTA TATAGC-3') were constructed by Guangzhou RiboBio Co., Ltd. The CD36-overexpression vector (Ov-CD36; $50 \mathrm{nM})$ and empty control vector (Ov-NC; $50 \mathrm{nM})$ were constructed by Shanghai GenePharma Co., Ltd. siRNA and plasmids were transfected into MLE-12 cells were inoculated into six-well plates $\left(5 \times 10^{5}\right.$ cells/well $)$, and then transfection was performed for $8 \mathrm{~h}$ at $37^{\circ} \mathrm{C}$ using Lipofectamine ${ }^{\circledR} 2000$ (Thermo Fisher Scientific, Inc.). After transfection for $48 \mathrm{~h}$, the transfection efficiency of cells in each group was detected via reverse transcription-quantitative (RT-q)PCR. Subsequent experiments were completed within $48 \mathrm{~h}$.

Reverse transcription quantitative PCR (RT-qPCR) analysis. $\mathrm{TRIzol}^{\circledR}$ reagent (Invitrogen; Thermo Fisher Scientific, Inc.) was used to extract total RNA from cells. Following which, $5 \mu \mathrm{g}$ total RNA was used as the control and cDNA was synthesized using AMV reverse transcription kit (Promega Corporation) according to the manufacturer's protocol. qPCR was performed on an ABI Prism 7900 Real-Time PCR system (Applied Biosystems; Thermo Fisher Scientific, Inc.) using a $\mathrm{SYBR}^{\circledR}$ Green kit (Qiagen, Inc.). The relative expression level of the target gene was calculated using the $2^{-\Delta \Delta \mathrm{Cq}}$ method (20). GAPDH was used for normalization. The following thermocycling conditions were used: Initial denaturation at $95^{\circ} \mathrm{C}$ for $10 \mathrm{~min}$; followed by 37 cycles of denaturation at $95^{\circ} \mathrm{C}$ for $15 \mathrm{sec}$ and annealing at $60^{\circ} \mathrm{C}$ for $1 \mathrm{~min}$; extension for $10 \mathrm{~min}$ at $65^{\circ} \mathrm{C}$. The sequences of PCR primers were as follows: CD36 Forward, 5'-AAGCCAGGTATTGCAGTTCTTT-3' and reverse, 5'-GCATTTGCTGATGTCTAGCACA-3'; STOM forward, 5'-GTGACTCTCGACAATGTAAC-3' and reverse, 5'-TGATCTCATAACGGAGGCAG-3'; and GAPDH forward, 5'-TGTCAAGCTCATTTCCTGGTAT-3' and reverse, 5'-CTC TCTTCCTCTTGTGCTCTTG-3'.

ELISA. Transfected cells were centrifuged at $1,000 \times \mathrm{g}$ for $5 \mathrm{~min}$ at $4^{\circ} \mathrm{C}$. The supernatant was collected and used for ELISA. The levels of malondialdehyde (MDA), superoxide dismutase (SOD), lactate dehydrogenase (LDH), TNF- $\alpha$ and ROS in the cell supernatant were measured using the MDA assay kit (cat. no. A003-1-2; Nanjing Jiancheng Bioengineering Institute), SOD Assay Kit (cat. no. A001-3-2; Nanjing Jiancheng Bioengineering Institute), LDH Cytotoxicity Assay Kit (cat. no. C0016; Beyotime Institute of Biotechnology), TNF- $\alpha$ assay kit (cat. no. H052-1; Nanjing Jiancheng Bioengineering Institute) and ROS assay kit (cat. no. S0033S; Beyotime Institute of Biotechnology), respectively, according to the manufacturer's protocol. These samples were measured using 

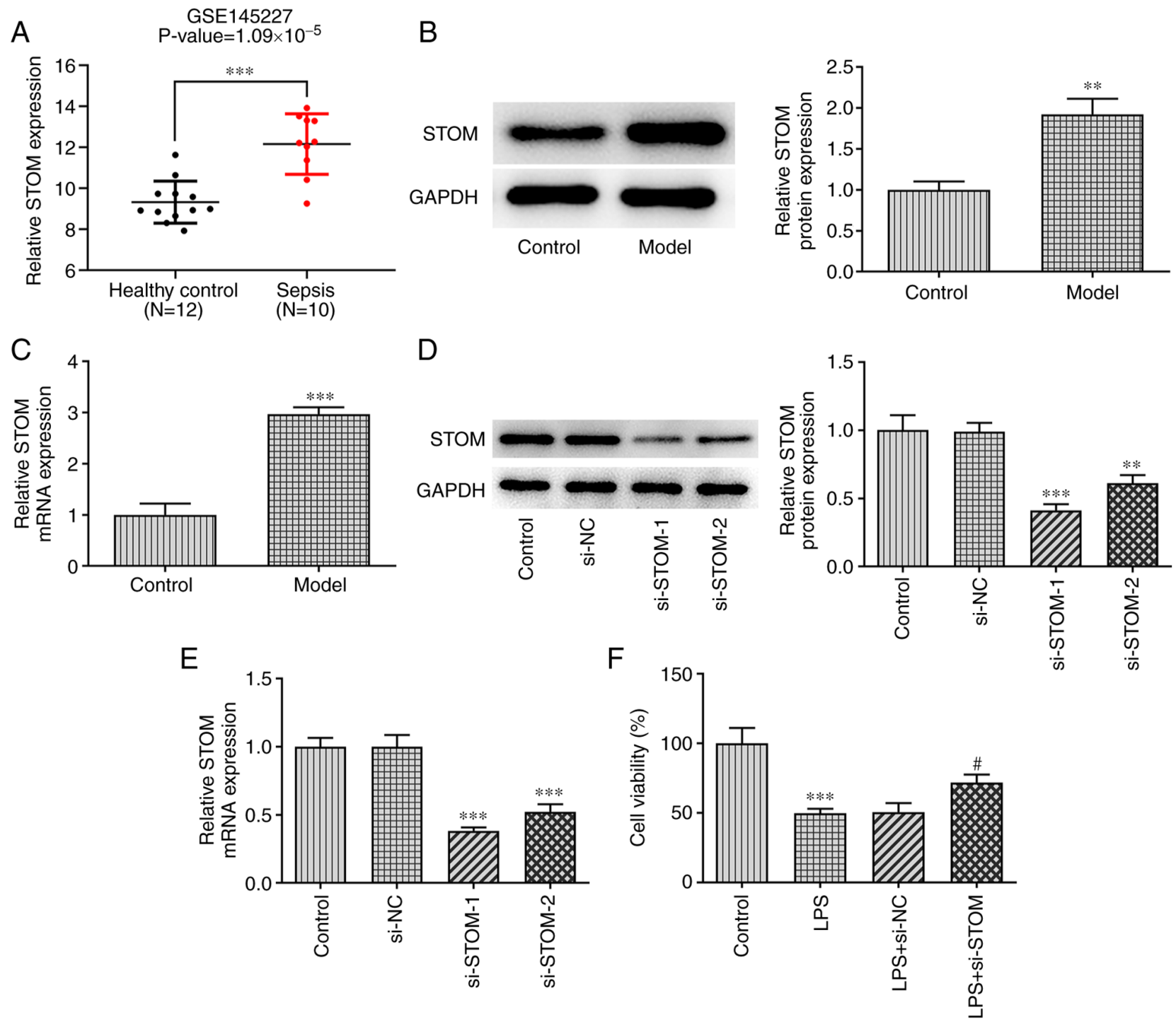

Figure 1. STOM expression in LPS-induced MLE-12 cells. (A) Gene expression of STOM was analyzed using the Gene Expression Omnibus database. ${ }^{* * *} \mathrm{P}<0.001$. Detection of STOM (B) protein and (C) mRNA expression levels in MLE-12 cells. (D) Detection of STOM protein and (E) mRNA expression levels in MLE-12 cells transfected with siRNA. (F) A Cell Counting Kit-8 assay was performed to determine cell viability. The results are representative of at least three independent experiments. ${ }^{* *} \mathrm{P}<0.01,{ }^{* * *} \mathrm{P}<0.001$ vs. Control group; ${ }^{*} \mathrm{P}<0.05$ vs. LPS + si-NC group. LPS, lipopolysaccharide; siRNA/si-, small interfering RNA; NC, negative control; STOM, stomatin.

a Hitachi spectrophotometer (F-7000 FL spectrophotometer; Hitachi, Ltd.).

Cell Counting Kit-8 (CCK-8) assay. Cells were inoculated on 96 -well plates at a density of $4 \times 10^{4}$ cells/well. The cells were cultured in DMEM/F12 medium containing 10\% FBS for 24,48 and $72 \mathrm{~h}$ at $37^{\circ} \mathrm{C}$. Subsequently, $10 \mu \mathrm{l}$ CCK-8 solution (cat. no. C0038; Beyotime Institute of Biotechnology) was added to each well to culture for $1 \mathrm{~h}$. The absorbance of cells in each well was detected at $450 \mathrm{~nm}$ using an automatic enzyme labeling instrument.

RNA immunoprecipitation (RIP) assay. The RIP assay was performed using the Magna RIP RNA-Binding Protein Immunoprecipitation kit (including RIP buffer and beads; cat. no. 17-700; MilliporeSigma) according to the manufacturer's protocol. Cells were incubated with RIP buffer supplied with the kit in an ice bath for $20 \mathrm{~min}$. A total of $20 \mu \mathrm{l}$ magnetic beads were applied for incubation of cell extracts and coated with $1 \mu \mathrm{g}$ Ago2 antibody (cat. no. 03-110; Merck KGaA) or $2 \mu \mathrm{g} \mathrm{IgG} \mathrm{anti-}$ body supplied with the kit for $12 \mathrm{~h}$ at $40^{\circ} \mathrm{C}$. Immunoprecipitated complexes were collected by adding $500 \mu \mathrm{l}$ lysate per $200 \mu \mathrm{l}$ RIP buffer, followed by centrifugation at $10,000 \mathrm{x} \mathrm{g}$ for $10 \mathrm{~min}$ at $4^{\circ} \mathrm{C}$. and RT-qPCR was subsequently performed.

Western blotting. Total protein was extracted from MLE-12 cells using RIPA lysis buffer (Beyotime Institute of Biotechnology). The protein concentration was detected using a BCA protein quantitative kit (Pierce; Thermo Fisher Scientific, Inc.). A total of $25 \mu \mathrm{g}$ protein per lane was separated via SDS-PAGE on a $10 \%$ gel, and subsequently transferred to PVDF membranes. These membranes were blocked with $5 \%$ non-fat milk for $2 \mathrm{~h}$ at room temperature. Membranes were incubated with primary antibodies $(1: 1,000)$ against STOM (cat. no. ab166623), CD36 (cat. no. ab133625), TNF- $\alpha$ (cat. no. ab215188), IL-6 (cat. no. ab233706) and GAPDH 

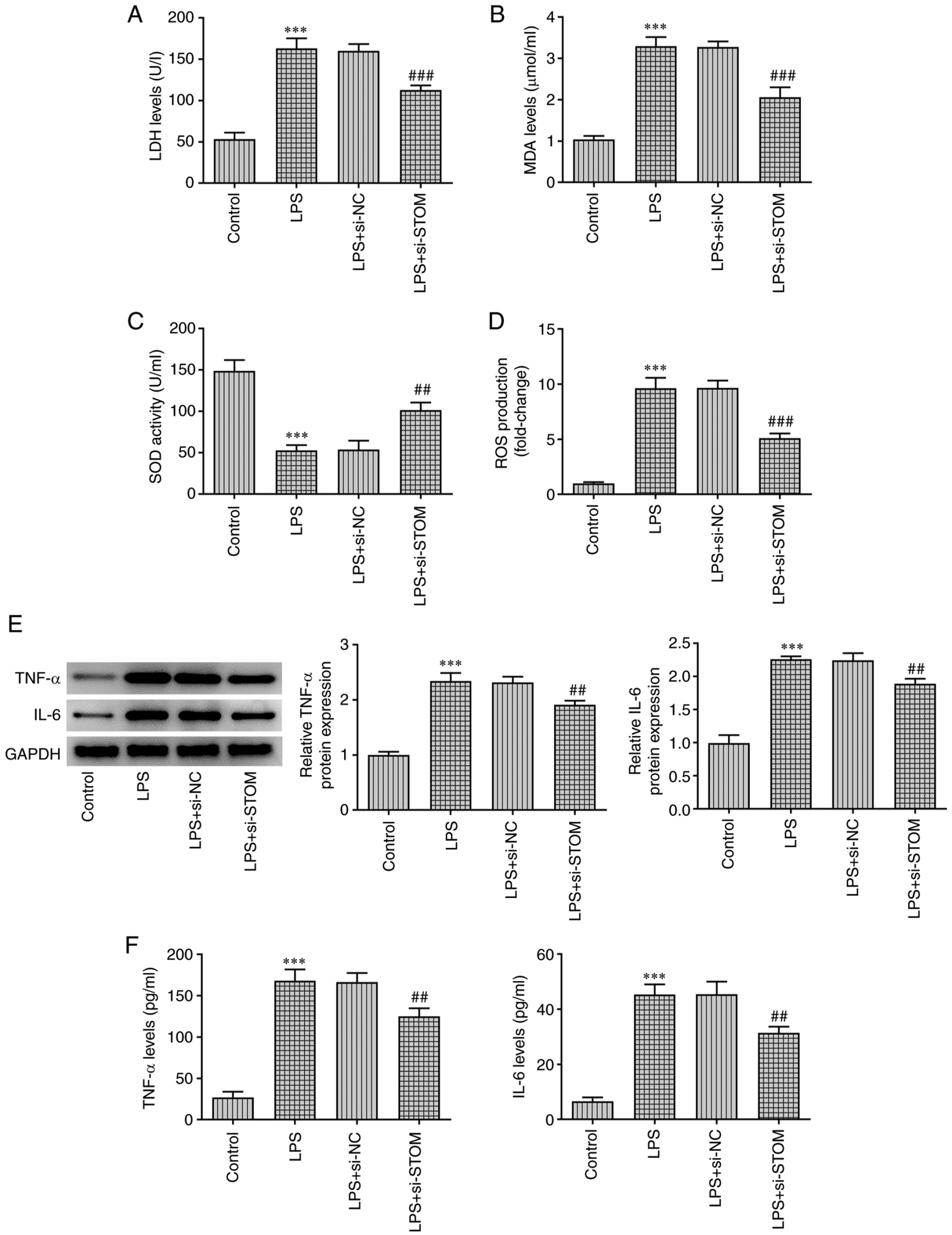

Figure 2. STOM-knockdown reduces oxidative stress and inflammation in LPS-induced MLE-12 cells. MLE-12 cells were treated with LPS, followed by transfection with si-NC and si-STOM. Detection of (A) LDH, (B) MDA, (C) SOD and (D) ROS levels in MLE-12 cells. Detection of TNF- $\alpha$ and IL-6 (E) protein expression levels and (F) content levels in MLE-12 cells. The results are representative of at least three independent experiments. ${ }^{* * *} \mathrm{P}<0.001$ vs. Control group; ${ }^{\# \#} \mathrm{P}<0.01,{ }^{\# \#} \mathrm{P}<0.001$ vs. LPS + si-NC group. LPS, lipopolysaccharide; si-, small interfering RNA; NC, negative control; LDH, lactate dehydrogenase; MDA, malondialdehyde; SOD, superoxide dismutase; ROS, reactive oxygen species; STOM, stomatin. 
A

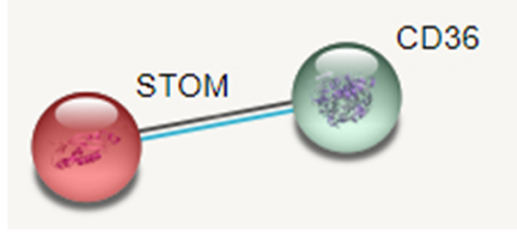

B

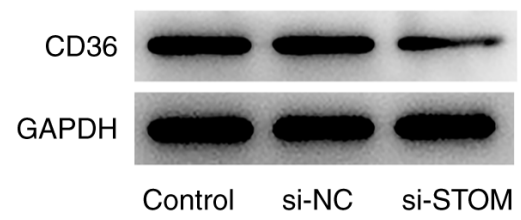

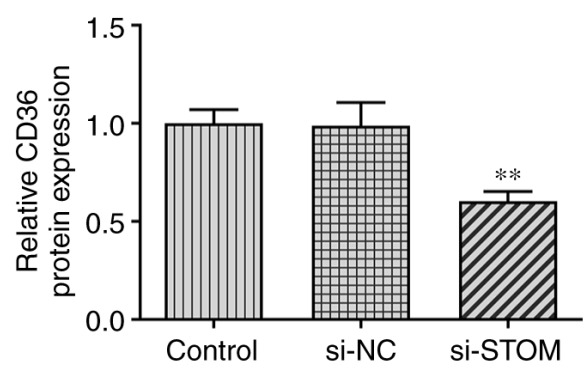

C

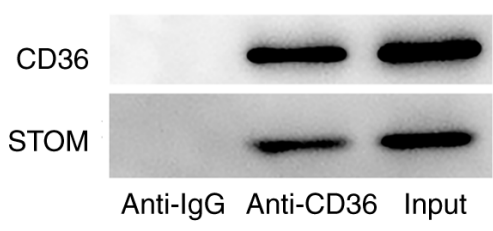

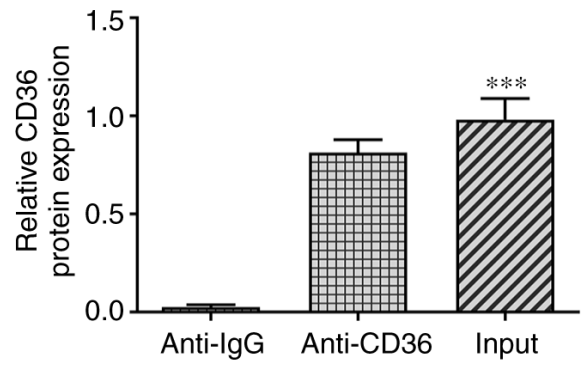

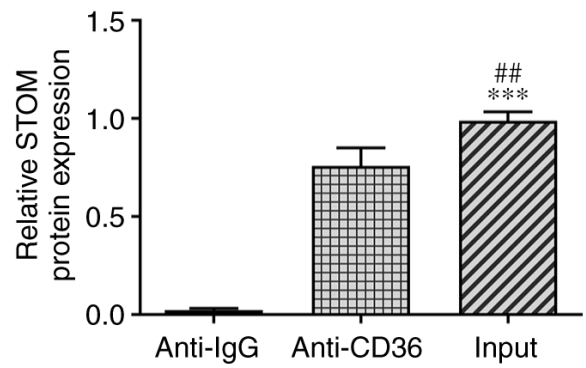

Figure 3. STOM positively regulates CD36. (A) Association between STOM and CD36 was predicted using the Search Tool for the Retrieval of Interacting Genes/Proteins database. (B) Detection of CD36 protein levels in MLE-12 cells transfected with si-STOM. ${ }^{* *}$ P $<0.01$ vs. Control group. (C) An RNA immunoprecipitation assay was conducted to determine the interaction between STOM and CD36. The results are representative of at least three independent experiments. ${ }^{* * *} \mathrm{P}<0.001$ vs. Anti-IgG group; ${ }^{\# \#} \mathrm{P}<0.01$ vs. Anti-CD36 group. si-, small interfering RNA; STOM, stomatin; NC, negative control.

(cat. no. ab9485) (all Abcam) overnight at $4^{\circ} \mathrm{C}$. After the membranes were washed with TBST $(0.1 \%$ Tween-20) three times, membranes were incubated with HRP-conjugated goat anti-rabbit (1:2,000; cat. no. 14708; Cell Signaling Technology, Inc.) secondary antibody for $2 \mathrm{~h}$ at room temperature. ECL reagent (Pierce; Thermo Fisher Scientific, Inc.) was used to detect the protein bands. ImageJ software 1.4.3 (National Institutes of Health) was used to detect the relative gray value of the target proteins.

Statistical analysis. GraphPad Prism 8.0 software (GraphPad Software, Inc.) was used for statistical analysis. The data are expressed as the mean \pm standard deviation (unless otherwise shown). One-way ANOVA followed by Tukey's post hoc test was used to analyze the comparison between multiple groups. An unpaired Student's t-test was used to compare differences between two groups. $\mathrm{P}<0.05$ was considered to indicate a statistically significant difference.

\section{Results}

STOM is upregulated in LPS-treated MLE-12 cells and STOM-knockdown promotes the viability of LPS-treated MLE-12 cells. GEO2R analysis of the GeneChip GSE145227 was employed to confirm that the expression level of STOM was significantly increased in the peripheral blood of newborns with sepsis compared with in the peripheral blood of healthy newborns (Fig. 1A). Subsequently, the results of RT-qPCR and western blotting demonstrated that the expression level of STOM in the sepsis model group was significantly higher compared with the control group (Fig. 1B and C). These results suggested that STOM may be involved in LPS-induced damage of MLE-12 cells.

The expression levels of STOM in MLE-12 cells were decreased following transfection with si-STOM.
As demonstrated in Fig. 1D and E, the expression levels of STOM in the si-NC group were similar to the control group; whereas STOM expression was decreased in both the si-STOM-1 and si-STOM-2 groups compared with the control group, with si-STOM-1 exhibiting an increased level of transfection efficiency compared with si-STOM-2. Therefore, si-STOM-1 was selected for follow-up experiments. A CCK-8 assay was applied to detect cell viability. As presented in Fig. 1F, the results demonstrated that treatment with LPS significantly inhibited cell viability compared with the control group, while STOM-knockdown reduced the inhibitory effect of LPS on cell viability compared with LPS+si-NC group.

STOM-knockdown reduces oxidative stress and inflammation in LPS-treated MLE-12 cells. Oxidative stress and inflammation levels were detected using ELISA and western blotting assays. As demonstrated in Fig. 2A-D, compared with the LPS + si-NC group, the levels of LDH, MDA and ROS in the LPS + si-STOM group were significantly decreased, whereas the level of SOD was significantly increased. Moreover, treatment with LPS induced high levels of LDH, MDA and ROS, and low levels of SOD compared with the control group. The results of western blotting and RT-qPCR analyses demonstrated that compared with the control group, LPS significantly increased the expression levels of the pro-inflammatory cytokines TNF- $\alpha$ and IL-6, whereas these changes were partially reversed by STOM-knockdown (Fig. 2E). Similar results were observed using ELISA. As presented in Fig. 2F, results of ELISA revealed that compared with the LPS + si-NC group, STOM-knockdown significantly decreased the levels of the pro-inflammatory cytokines TNF- $\alpha$ and IL-6. These results indicated that STOM-knockdown reduced oxidative stress and inflammation in MLE-12 cells treated with LPS. 
A

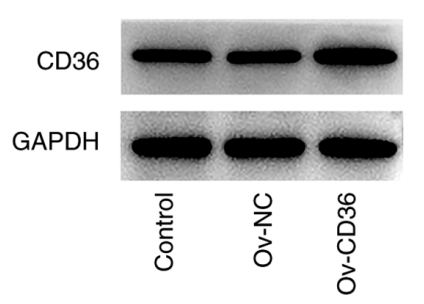

C

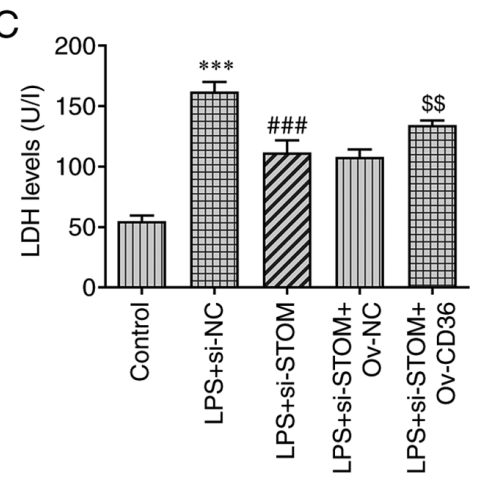

$\mathrm{F}$
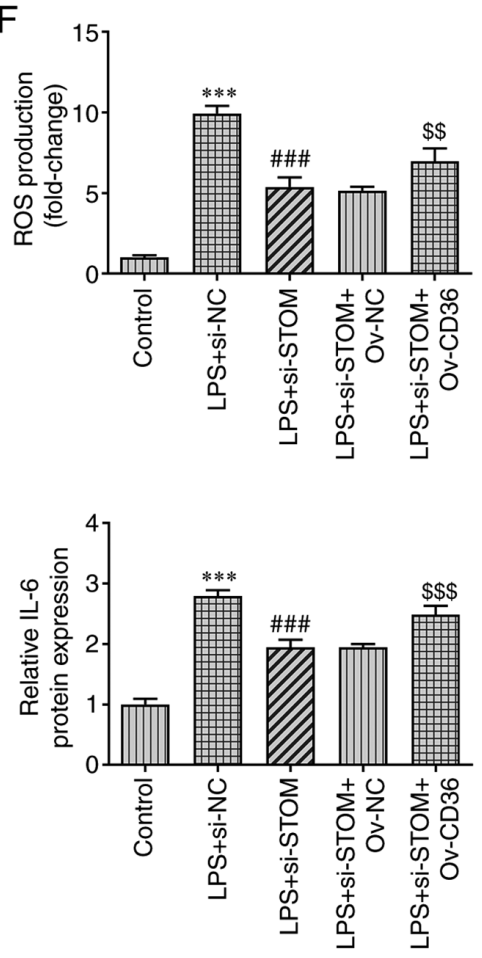
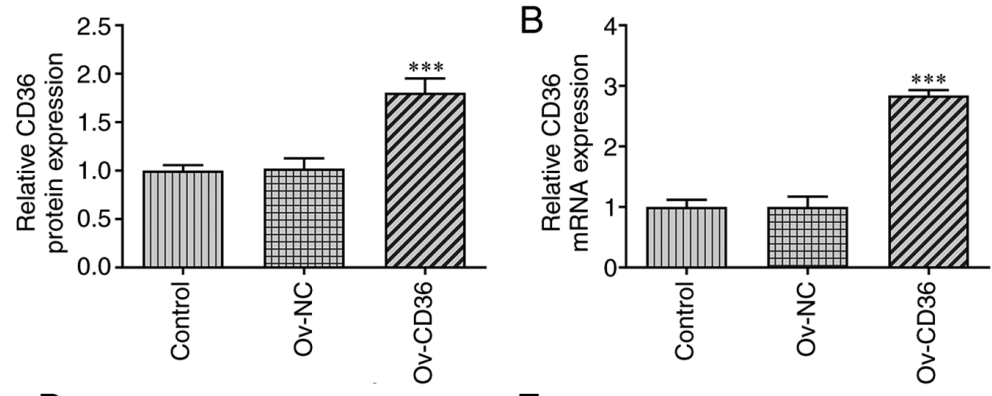

E

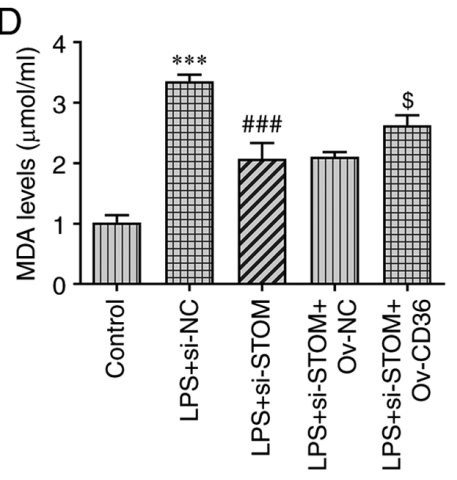

G
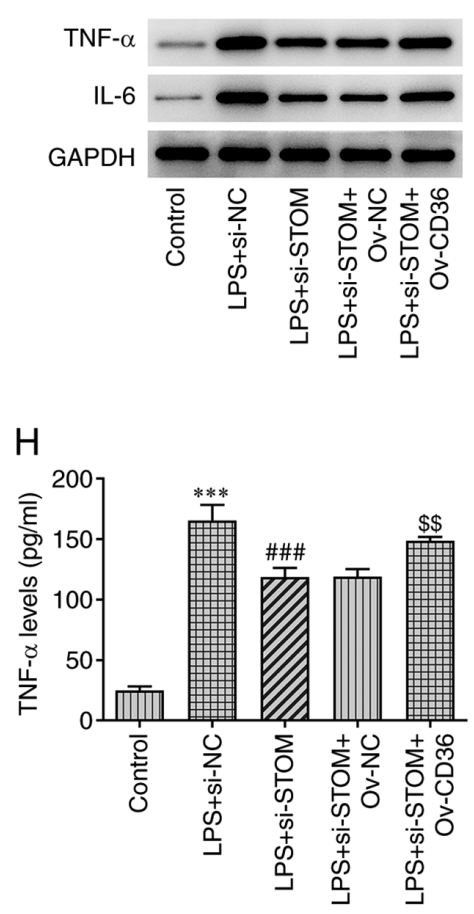
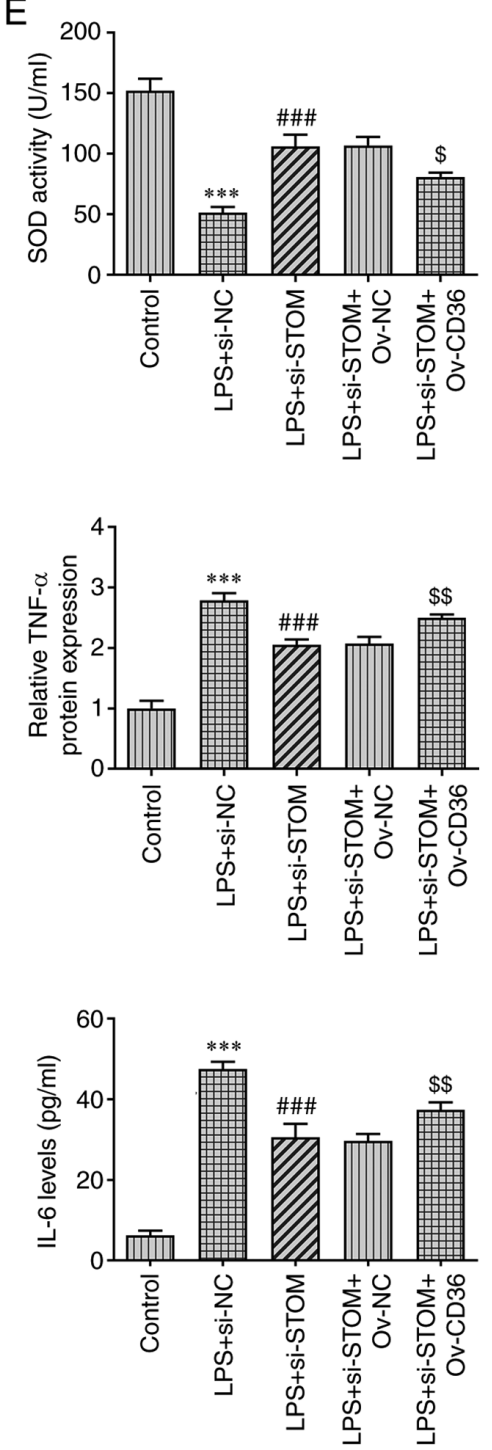

Figure 4. STOM promotes oxidative stress and inflammation in LPS-induced MLE-12 cells. Detection of CD36 (A) protein and (B) mRNA levels in MLE-12 cells transfected with Ov-NC and Ov-CD36. Detection of (C) LDH, (D) MDA, (E) SOD and (F) ROS levels in LPS-induced MLE-12 cells transfected with Ov-NC and Ov-CD36. Detection of TNF- $\alpha$ and IL-6 (G) protein and (H) mRNA levels in MLE-12 cells. The results are representative of at least three independent experiments. ${ }^{* * *} \mathrm{P}<0.001$ vs. Control group; ${ }^{\# \# t} \mathrm{P}<0.001$ vs. $\mathrm{LPS}+\mathrm{si}-\mathrm{NC}$ group; ${ }^{{ }^{\circ}} \mathrm{P}<0.05,{ }^{\$ \$} \mathrm{P}<0.01,{ }^{\$ \$ \$} \mathrm{P}<0.001$ vs. LPS + si-STOM $+\mathrm{Ov}-\mathrm{NC}$ group. LPS, lipopolysaccharide; Ov-, overexpression; NC, negative control; LDH, lactate dehydrogenase; MDA, malondialdehyde; SOD, superoxide dismutase; ROS, reactive oxygen species; si-, small interfering RNA; STOM, stomatin.

STOM positively regulates CD36. To further explore the effects of STOM on the damage of MLE-12 cells treated with LPS, the downstream target gene of STOM, CD36, was screened for using the STRING database (Fig. 3A). The interaction between STOM and CD36 was verified using a RIP assay (Fig. 3C). The results revealed that CD36 and STOM bound strongly. Moreover, as demonstrated in Fig. 3B, STOM-knockdown significantly decreased the expression of CD36 compared with the control. Thus, results of the present study demonstrated that STOM positively regulated CD36.

STOM promotes oxidative stress and inflammation in LPS-treated MLE-12 cells by upregulating CD36. Lastly, a CD36-overexpressing plasmid (Ov-CD36) was constructed. As displayed in Fig. 4A and B, compared with the Ov-NC group, both the protein and mRNA expression levels of CD36 
were significantly increased in the Ov-CD36 group. Detection of oxidative stress levels using the kits revealed a significant decrease in LDH levels after interfering with STOM compared with the LPS + si-NC group. However, the inhibitory effect of interfering with STOM on LDH was reversed after CD36 overexpression (Fig. 4C). Additionally, the trend of MDA and ROS among groups was similar to that of LDH (Fig. 4D and F). By contrast, following interference with STOM, SOD levels were upregulated compared with the LPS + si-NC group, but following CD36 overexpression, SOD levels were decreased (LPS + si-STOM + Ov-CD36 vs. LPS + si-STOM + Ov-NC; Fig. 4E). As demonstrated in Fig. 4C-F, the overexpression of CD36 reversed the inhibitory effect of STOM-knockdown on oxidative stress. As demonstrated in Fig. 4G and H, TNF- $\alpha$ and IL-6 expression was suppressed after knockdown of STOM compared with the LPS + si-NC group. However, overexpression of CD36 significantly increased TNF- $\alpha$ and IL-6 expression compared with the knockdown of STOM group. Therefore, it was clear that overexpression of CD36 alleviated the inhibitory effect of STOM-knockdown on inflammation. Collectively, these results demonstrated that STOM aggravated LPS-induced damage of MLE-12 cells by promoting the expression of CD36.

\section{Discussion}

Alveolar epithelial cells act as important barriers against external pathogens and are closely associated with the occurrence and development of lung diseases, such as lung injury and lung fibrosis (21). High levels of oxidative stress and inflammation in alveolar epithelial cells destroy the integrity of lung alveolar epithelial cells and induce ALI (22). Bacterial endotoxin LPS is the main component of the outer wall of Gram-negative bacteria, which promotes apoptosis and induces ALI (23). In the present study, STOM-knockdown significantly suppressed LPS-induced oxidative stress and inflammation in MLE-12 cells by downregulating the expression level of CD36. These results suggested that STOM may play a notable role in LPS-induced MLE-12 cell injury.

STOM is a unidirectional lipid raft-related protein isolated from the plasma membrane of normal human erythrocytes (24). To date, there is relatively limited direct research on the function of STOM. The analysis of GeneChip GSE145227 of neonatal patients with sepsis demonstrated that, compared with healthy newborns, the expression levels of STOM in the peripheral blood of newborns with sepsis were significantly increased (25). In addition, STOM is highly expressed in alveolar epithelial cells treated under hypoxic conditions (10), and oxidative stress and inflammation are caused by hypoxia (14). STOM-like protein 2 (SLP-2) is one of the most widely studied homologs of STOM. Studies have reported that SLP-2 is upregulated in a variety of cancer types, including esophageal squamous cell carcinoma (26), epithelial ovarian cancer (27) and non-small cell lung cancer, amongst others (28), and that SLP-2-silencing significantly reduces cancer progression (29). Notably, SLP-2 acts as a pro-inflammatory factor in patients with colitis and liver cancer $(30,31)$. Thus, STOM may affect the oxidative stress and inflammation of alveolar epithelial cells induced by sepsis. In the present study, STOM was highly expressed in LPS-treated MLE-12 cells compared with the control group. STOM-knockdown significantly enhanced the viability of LPS-treated MLE-12 cells, and suppressed oxidative stress and inflammation, compared with the LPS-treated group. These results suggested that STOM promoted oxidative stress and inflammation in LPS-induced MLE-12 cells.

The effects of STOM on sepsis-induced MLE-12 cell injury were further explored, and results obtained from the STRING database demonstrated that CD36 interacts with STOM. CD36 is a single-stranded transmembrane cell surface protein widely found in various cells, including monocytes, macrophages and microvascular endothelial cells $(15,32)$. CD36 plays a key role in the transport of fatty acids in the liver by participating in the transmembrane transport of long-chain fatty acids (33). A previous study reported that CD36 promotes the inflammation of macrophages and microglia by binding to toll-like receptors (34). Notably, CD36-silencing effectively reduces LPS-induced inflammation and ALI (25). The results of the present study demonstrated that STOM positively regulated the expression level of CD36. Furthermore, overexpression of CD36 partially reversed the promoting effects of STOM-knockdown on oxidative stress and inflammation in LPS-treated MLE-12 cells. There are some limitations to the present study, such as the lack of multiple cell lines used to validate the findings, and likewise, the lack of in vivo experiments.

In conclusion, the expression levels of STOM in mouse alveolar epithelial cells treated with LPS were significantly increased compared with those in the control group, and STOM-knockdown reduced the levels of oxidative stress and inflammation by binding to CD36 in ALI. These results preliminarily confirmed the role of STOM to be a valuable biomarker in the progression of ALI. Thus, results obtained during the present study may provide a theoretical basis for understanding the regulatory mechanisms underlying ALI, and provide support for the development of novel treatment options for ALI in the future. Moreover, further in vitro experiments will be carried out using alternative cell lines to MLE-12, and these will be supplemented with in vivo experiments, to further confirm the role of STOM in ALI in future investigations.

\section{Acknowledgements}

Not applicable.

\section{Funding}

No funding was received.

\section{Availability of data and materials}

The datasets used and/or analyzed during the current study are available from the corresponding author on reasonable request.

\section{Authors' contributions}

Both KW and LW were involved in the protocol design and experiments used in the present study. All authors were responsible for analysis and interpretation of data. KW was responsible for writing and editing the manuscript. $\mathrm{KW}$ and 
LW confirm the authenticity of all the raw data. All authors have read and approved the final manuscript.

\section{Ethics approval and consent to participate}

Not applicable.

\section{Patient consent for publication}

Not applicable.

\section{Competing interests}

The authors declare that they have no competing interests.

\section{References}

1. Singer M, Deutschman CS, Seymour CW, Shankar-Hari M, Annane D, Bauer M, Bellomo R, Bernard GR, Chiche JD, Coopersmith CM, et al: The third international consensus definitions for sepsis and septic shock (Sepsis-3). JAMA 315: 801-810, 2016.

2. Salomao R, Ferreira BL, Salomão MC, Santos SS, Azevedo LCP and Brunialti MKC: Sepsis: Evolving concepts and challenges. Braz J Med Biol Res 52: e8595, 2019.

3. Fleischmann C, Scherag A, Adhikari NK, Hartog CS, Tsaganos T, Schlattmann P, Angus DC and Reinhart K; International Forum of Acute Care Trialists: Assessment of global incidence and mortality of hospital-treated sepsis. Current estimates and limitations. Am J Respir Crit Care Med 193: 259-272, 2016.

4. Thompson BT, Chambers RC and Liu KD: Acute respiratory distress syndrome. N Engl J Med 377: 562-572, 2017.

5. Sawa T: The molecular mechanism of acute lung injury caused by pseudomonas aeruginosa: From bacterial pathogenesis to host response. J Intensive Care 2: 10, 2014.

6. Bellani G, Laffey JG, Pham T, Fan E, Brochard L, Esteban A, Gattinoni L, van Haren F, Larsson A, McAuley DF, et al: Epidemiology, patterns of care, and mortality for patients with acute respiratory distress syndrome in intensive care units in 50 countries. JAMA 315: 788-800, 2016.

7. Stewart GW: Stomatin. Int J Biochem Cell Biol 9: 271-274, 1997.

8. Genetet S, Desrames A, Chouali Y, Ripoche P, Lopez C and Mouro-Chanteloup I: Stomatin modulates the activity of the anion exchanger 1 (AE1, SLC4A1). Sci Rep 7: 46170, 2017.

9. Nagarajan A, Dogra SK, Sun L, Gandotra N, Ho T, Cai G, Cline G, Kumar P, Cowles RA and Wajapeyee N: Paraoxonase 2 facilitates pancreatic cancer growth and metastasis by stimulating GLUT1-mediated glucose transport. Mol Cell 67: 685-701. e686, 2017.

10. Chen JC, Cai HY, Wang Y, Ma YY, Song LN, Yin LJ, Cao DM, Diao F, Li YD and Lu J: Up-regulation of stomatin expression by hypoxia and glucocorticoid stabilizes membrane-associated actin in alveolar epithelial cells. J Cell Mol Med 17: 863-872, 2013.

11. Wang D, Qi H, Li A, Deng F, Xu Y, Hu Z and Liu Q: Coexisting overexpression of STOML1 and STOML2 proteins may be associated with pathology of oral squamous cell carcinoma. Oral Surg Oral Med Oral Pathol Oral Radiol 129: 591-599.e593, 2020.

12. Long $G$ and Yang C: A sixgene support vector machine classifier contributes to the diagnosis of pediatric septic shock. Mol Med Rep 21: 1561-1571, 2020.

13. Wang M, Li C and Shi W: Stomatin-like protein-2 confers neuroprotection effect in oxygen-glucose deprivation/ reoxygenation-injured neurons by regulating AMPK/Nrf2 signalling. J Drug Target 28: 600-608, 2020.

14. McGarry T, Biniecka M, Veale DJ and Fearon U: Hypoxia, oxidative stress and inflammation. Free Radic Biol Med 125: 15-24, 2018.
15. Wang $\mathrm{J}$ and Li Y: CD36 tango in cancer: Signaling pathways and functions. Theranostics 9: 4893-4908, 2019.

16. Banesh S and Trivedi V: Therapeutic potentials of scavenger receptor cd36 mediated innate immune responses against infectious and non-infectious diseases. Curr Drug Discov Technol 17: 299-317, 2020

17. Bocharov AV, Wu T, Baranova IN, Birukova AA, Sviridov D, Vishnyakova TG, Remaley AT, Eggerman TL, Patterson AP and Birukov KG: Synthetic amphipathic helical peptides targeting CD36 attenuate lipopolysaccharide-induced inflammation and acute lung injury. J Immunol 197: 611-619, 2016.

18. Li $\mathrm{J}$ and Liu S: LncRNA GAS5 suppresses inflammatory responses and apoptosis of alveolar epithelial cells by targeting miR-429/DUSP1. Exp Mol Pathol 113: 104357, 2020.

19. Bai Z, Li Y, Li Y, Pan J, Wang J and Fang F: Long noncoding RNA and messenger RNA abnormalities in pediatric sepsis: A preliminary study. BMC Med Genomics 13: 36, 2020.

20. Livak KJ and Schmittgen TD: Analysis of relative gene expression data using real-time quantitative PCR and the 2(-Delta Delta C(T)) method. Methods 25: 402-408, 2001.

21. Shao L, Meng D, Yang F, Song H and Tang D: Irisin-mediated protective effect on LPS-induced acute lung injury via suppressing inflammation and apoptosis of alveolar epithelial cells. Biochem Biophys Res Commun 487: 194-200, 2017.

22. Hu Q, Wang Q, Han C and Yang Y: Sufentanil attenuates inflammation and oxidative stress in sepsis-induced acute lung injury by downregulating KNG1 expression. Mol Med Rep 22: 4298-4306, 2020.

23. Zeng M, Huang $\mathrm{C}$, Zheng $\mathrm{H}$, Chen $\mathrm{Q}, \mathrm{He} \mathrm{W}$ and Deng $\mathrm{Y}$ : Effects of ghrelin on iNOS-derived NO promoted LPS-induced pulmonary alveolar epithelial A549 cells apoptosis. Cell Physiol Biochem 49: 1840-1855, 2018.

24. Basu A, Harper S, Pesciotta EN, Speicher KD, Chakrabarti A and Speicher DW: Proteome analysis of the triton-insoluble erythrocyte membrane skeleton. J Proteomics 128: 298-305, 2015.

25. Li Y, Li Y, Bai Z, Pan J, Wang J and Fang F: Identification of potential transcriptomic markers in developing pediatric sepsis: A weighted gene co-expression network analysis and a case-control validation study. J Transl Med 15: 254, 2017.

26. Cao W, Zhang B, Ding F, Zhang W, Sun B and Liu Z: Expression of SLP-2 was associated with invasion of esophageal squamous cell carcinoma. PLoS One 8: e63890, 2013.

27. Guo XY, Guo HF and Guo HM: Clinical significance of SLP-2 in epithelial ovarian cancer and its regulatory effect on the notch signaling pathway. Eur Rev Med Pharmacol Sci 24: 1666-1671, 2020.

28. Yang CT, Li JM, Li LF, Ko YS and Chen JT: Stomatin-like protein 2 regulates survivin expression in non-small cell lung cancer cells through $\beta$-catenin signaling pathway. Cell Death Dis 9: 425, 2018.

29. Zhou C, Li Y, Wang G, Niu W, Zhang J, Wang G, Zhao Q and Fan L: Enhanced SLP-2 promotes invasion and metastasis by regulating Wnt/ $\beta$-catenin signal pathway in colorectal cancer and predicts poor prognosis. Pathol Res Pract 215: 57-67, 2019.

30. Kucuk I, Tanoglu A, Öncü K, YılmazI,Kara M, Beyazıt Y,Akyol T, Kaplan M, Ozarı HO and Yazgan Y: Immunohistochemical activity of prohibitin-2 and stomatin-like protein-2 in patients with ulcerative colitis. Turk J Gastroenterol 27: 233-238, 2016.

31. Pu X, Dong C, Zhu W, Li W and Jiang H: Silencing stomatin-like protein 2 attenuates tumor progression and inflammatory response through repressing CD14 in liver cancer. Onco Targets Ther 12: 7361-7373, 2019.

32. Park YM: CD36, a scavenger receptor implicated in atherosclerosis. Exp Mol Med 46: e99, 2014.

33. Pepino MY, Kuda O, Samovski D and Abumrad NA: Structure-function of CD36 and importance of fatty acid signal transduction in fat metabolism. Annu Rev Nutr 34: 281-303, 2014.

34. Li X, Zhang X, Pang L, Yao L, ShangGuan Z and Pan Y: Agaricus bisporus-derived $\beta$-glucan enter macrophages and adipocytes by CD36 receptor. Nat Prod Res 34: 3253-3256, 2020.

This work is licensed under a Creative Commons Attribution-NonCommercial-NoDerivatives 4.0 International (CC BY-NC-ND 4.0) License. 